\title{
High-resolution bottom-up residential electrical model for distribution networks planning
}

\author{
Simon Sansregret ${ }^{1}$, Karine Lavigne ${ }^{1}$, Brice Le Lostec ${ }^{1}$, François Laurencelle ${ }^{1}$, Frédéric Guay ${ }^{2}$ \\ ${ }^{1}$ Laboratory of Energy Technologies, Hydro-Québec Research Institute, Shawinigan, Canada \\ ${ }^{2}$ Hydro-Québec Research Institute, Varennes, Canada
}

\begin{abstract}
This paper proposes a bottom-up residential electrical model to predict the electric load profile on a regional scale under different scenarios of evolution. The model, which simulates occupant behavior diversity, aims to support research in a wide range of fields, including demand-side management, load shifting and the impact of rate signals. The proposed methodology was applied to 962 electrically heated homes of electric utility customers located in Quebec (Canada). Preliminary results show limited gaps between aggregated simulated load profiles and measured ones, giving confidence to extend the development of this kind of model to distribution networks planning.
\end{abstract}

\section{Introduction}

Load forecasting is essential for electricity distribution network planning. It helps utilities make decisions on power management, load switching, network reconfiguration and infrastructure development. In a context of growth in electric heating demand and the availability of distributed energy resources, such as solar, energy storage and demand response measures, the load curve will definitely be impacted, increasing the challenge and importance of load forecasting. Electric vehicles, photovoltaics, batteries, incentive rates for shifting consumption outside peak periods, urbanization, and behavioral changes (e.g. people who work at home) are just a few examples that could have a significant impact on electrical loads and could challenge current load forecasting methods.

Kuster (2017) presents an interesting literature review of 113 electrical load forecasting models. Most of the methods for medium- and long-term demand forecasting correlate historical measured profiles with independent variables such as weather and socio-economic indices, using either time series techniques or neural network approaches. These methods may fail to address the effects of specific measures, new technology penetration or behavioral change, since they rely on past observation. A small number of methods have risen in past years using a bottom-up approach in the residential sector. They involve the stochastic aggregation of enduse load predictions based on occupants' activities and appliance utilization patterns. This is the approach the authors have explored because of its great flexibility.

In this paper, a stochastic model based on a bottom-up approach is used to predict residential electric load profiles on a regional scale. The dwelling characteristics, appliances and occupant attributes are statistically derived from sets of open data for specific regions. Stochastic profiles of presence, activities and appliance use related to occupant attributes are obtained from models based on information from general social timeof-use surveys (TUS). Finally, simulated electric appliances profiles of buildings in the target region are obtained from specific appliance models, at one-minute intervals, and compared with utility data to improve and calibrate the models.

The focus of the approach is to simulate the behavioral aspect, which has a large impact on the load profile and its variation. It explains the load profile as an aggregation of end-use appliance consumption, which depends on household activities and behavior. This could support research in demand-side management, load shifting and the impact of rate signals, for example.

A brief overview of the literature on bottom-up models focusing on behavior is provided, as cited also by Vorger (2014), Aerts (2015), Fischer (2015) and Wills (2017). A complete review has been done recently by Happle (2018).

Capasso (1994) presents a bottom-up model of the electricity demand of a residential building stock in Italy. The domestic activities are modelled from demographic and socio-economic data. Appliance power consumption is computed based on unit consumption profiles triggered by activities. The profiles are then aggregated to simulate the building stock.

Richardson (2008) (2010) also presents a bottom-up model for building stock modelling (CREST model), which is based on a stochastic approach. A behavioral model is designed with TUS data. Appliance activation depends on this behavioral model and is calibrated to represent typical annual consumption by end-use. Again, the aggregated profile simulates the building stock. It should be noted that socio-economic characteristics are not considered in his approach.

Fischer (2015) presents another approach similar to Richardson (2008) (2010) to model the power demand profile of a building stock. The proposed methodology integrates socio-economic criteria and seasonal variations in appliance use, based on data samples with a high time-resolution.

Wilke (2013) and Vorger (2014) present similar methods to model a residential electric load profile. These are based on the fact that people's demographic and socio- 
economic characteristics impact the power consumption of their home. The dwellings in the model are therefore populated in such a way as to represent the population's characteristics. A stochastic behavioral model dependent on these characteristics is developed from TUS data. It establishes operation profiles and power demand profiles of appliances and of the building stock. The distribution of appliances in the dwellings still represents the market.

The model proposed by the present authors benefits from previous works as explained in more detail in the following sections. Extensive sets of open data and the availability of more than 10,000 electric residential profiles at 15-minute intervals from utility meters provide interesting opportunities for exploring this approach. The methodology used by the authors results from a brief proof of concept, and future works are presented below.

\section{Methodology}

The general approach to generating domestic load profiles on a regional scale consists of three steps, as shown in Figure 1.
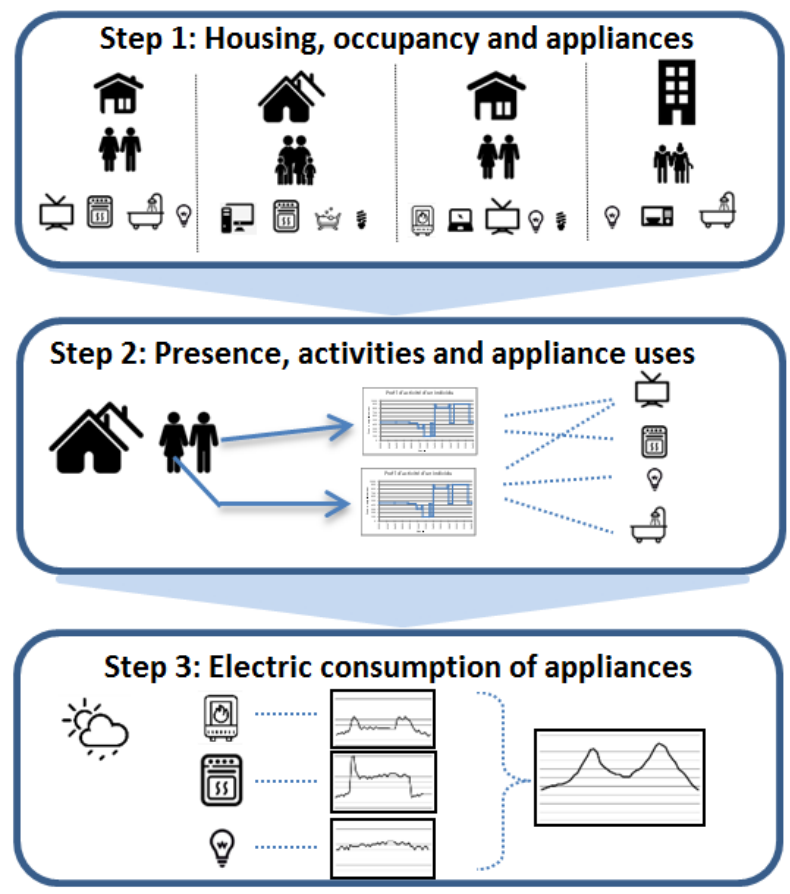

Figure 1: Steps in the approach

The first step generates occupied dwellings with specific appliances located in the geographical region of interest. The attributes of the dwellings (e.g., surface area, year of construction), occupancy (e.g., household size, age, status) and appliances (e.g., presence, type, efficiency) are statistically derived from various sources of data characterizing the region. The second step assesses a profile of absence/presence and activities of each occupant according to their attributes. Based on these activities, appliance use profiles are derived. Finally, in the last step, the electricity consumption of each appliance in all occupied dwellings of the region is estimated by different models. The following sections provide details on each step.

\section{Housing, occupancy and appliances}

The first step in the modeling consists in allocating the characteristics of the dwelling, household and electrical equipment/appliances to each house in the modeled stock. Two possible approaches were identified (Vorger, 2014; Ansanay-Alex, 2016) to carry out this first step. Vorger (2014) presents a population model where mandatory input data include the type of building, its location and the number of rooms. The characteristics of the household and its occupants are then determined sequentially based on survey data with conditional probabilities among certain of the attributes. The household population approach of Ansanay-Alex (2016) is based on a Bayesian network that models dwelling characteristics on the joint distribution of national census variables.

For our work, the Ansanay-Alex (2016) method was selected because it has more flexibility compared to the approach proposed by Vorger (2014). Bayesian network models were developed on different territorial scales (national, provincial, census agglomerations) to generate the population of dwellings, which includes the attributes of dwellings, households, and equipment/appliances. Data from Census Canada (Statistics Canada, 2016) is used for attributes affecting dwellings and households. Data from Canada's 2009 Survey of Household Spending (Statistics Canada, 2009) is used to populate the dwellings with household appliances. Figure 2 shows a simplified example of such a network.

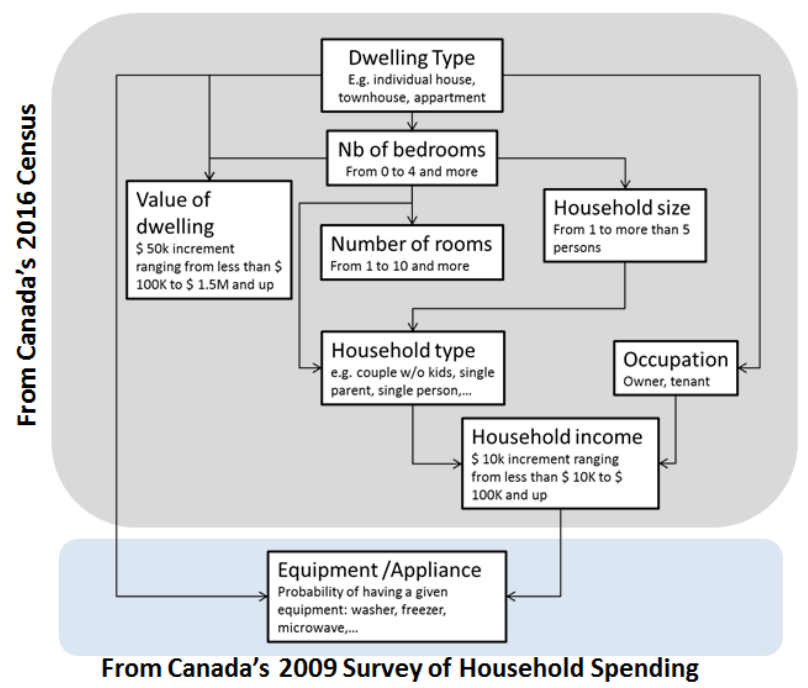

Figure 2: Example of Bayesian network

The advantage of a Bayesian network is that it can be used to impose constraints on certain attributes. Figure 3 shows a simplified example of a Bayesian network where a constraint on the type of construction was applied. As shown, the distributions of the other attributes were recalculated.

For each dwelling modeled in the building stock, it is possible to extract the type of construction and the corresponding census agglomeration from the property assessment roll. The latter makes it possible to identify the proper Bayesian network, while the former applies a 
constraint on the dwelling type in order to derive the probabilities for the other attributes.
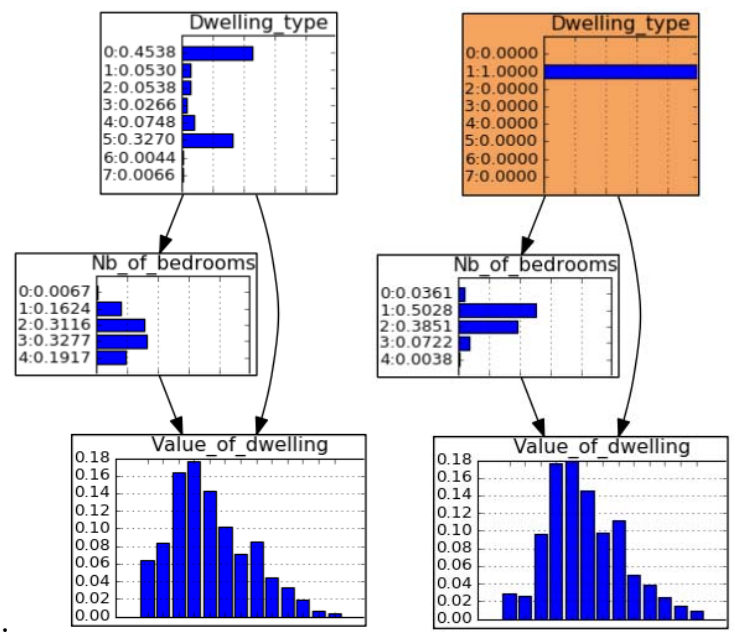

Figure 3: Example of simplified Bayesian network with (right) and without (left) constraint

The Bayesian network developed was used to represent attributes of a certain number of households, and the results were compared to survey data to verify their validity. For example, Figure 4 shows the distribution of the number of televisions per household in a building stock of 100 dwellings. The distribution obtained with the Bayesian network shows good agreement with the survey data.

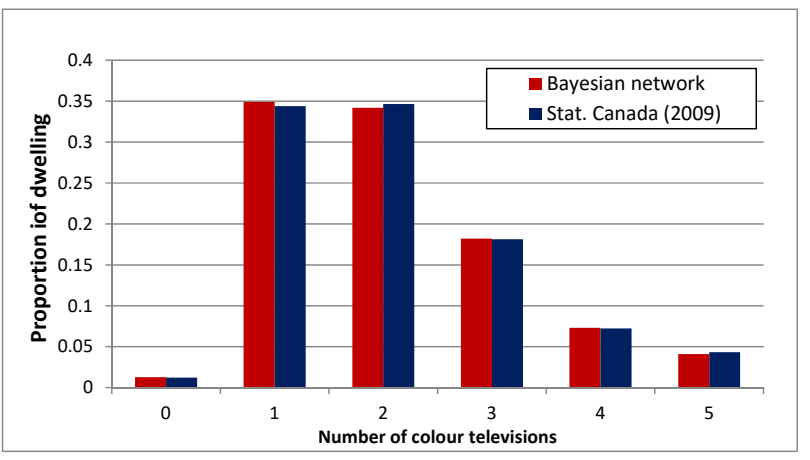

Figure 4: Example of comparison between Bayesian network results and survey data

The strategy used to determine some missing attributes - essentially the floor areas of individual rooms and their type (kitchen, bedroom...) - is based on a sample of 30 properties whose attributes have been verified. These houses have been separated into three archetypes (11 bungalows, 11 split-levels and 8 cottages). For each archetype, the probability of presence of each room type is determined. In addition, statistic distributions of floor area and installed heating power per area were calculated for 12 identified room types.

\section{Presence, activities and appliance use}

Once the characteristics of the dwellings, households and electrical appliances are determined, the next step consists in simulating household behavior and appliance use profiles. The simulation of the behavior of the individual occupants or the household was based on the hypothesis reported by Page (2008) where the presence of individuals in a dwelling is a condition to interact with the building. Even if this hypothesis is somewhat restrictive-because home automation systems, timers and remote controls could invalidate them (robot vacuum cleaners, programmable decoders, blinds, lighting, etc.) - the fact remains that the internal gains (release of heat/humidity) and most activities in a house still require the presence of individuals (using the shower, for instance). Several behavioral models use a stochastic approach based on TUS, since the models represent behaviors individualized according to the occupants' characteristics and to cultural and geographic specificities. Consequently, this particular type of model was selected for our study.

Capasso (1994) proposes a statistical model based on the Monte Carlo method to activate appliances when the home is occupied. The presence profile is based on typological methods (for example, no human presence from 8 a.m. to noon and from 4 p.m. to 6 p.m.). To account for certain variability in the presence profile, Capasso proposed using a Monte Carlo method to determine the hours of departure and arrival based on an average profile. These hours included the occupants' activities and were divided into four categories: household chores, personal hygiene, cooking and entertainment. The authors associated each category with a distribution of probabilities as a function of time over a day. Based on these elements, Capasso defined a presence profile in conjunction with an activity profile. The two profiles demonstrate the probability that an individual will use a particular appliance associated with his or her activity profile. Paatero and Lund (2006) used a similar method, but in theirs it is not possible to represent the diversity of an occupation profile since it is based on the average typological profile of a given presence model.

Wilke (2013) and Vorger (2014) present an individualized presence model (active or inactive) based on the Markov process, which is similar to the Richardson (2008) approach. Nevertheless, a presence model is applied to each of the household's occupants. Transition matrices determined based on TUS are applied to each individual. At the beginning of an individual's presence, his or her activity and its duration are determined. When the activity ends before the end of the individual's presence, a new activity begins. This activity is interrupted at the end of the presence period. Therefore, the models proposed by Wilke (2013) or Vorger (2014) take into account the duration of activities as well as the arrival or departure of the individual occupants. This type of method has the disadvantage of allowing a significant fluctuation in the number of individuals who are present in the dwelling at a given time, which can have an effect on activities and, by the same token, on the power consumption of electrical appliances. Furthermore, as noted by Vorger (2014), the presence and activity models are independently generated for each individual whereas, intuitively, it would be more realistic to also generate household scenarios since certain activities, meals for instance, 
generally take place at the same time and involve all the individuals. To deal with this significant presence fluctuation when using the Markov process in the order of 1, Wilke (2013) also presents a high-order Markov method. When a presence begins, its duration is determined based on a probability distribution depending on the day and the time of the beginning of the presence. Presence models similar to Wilke's are also presented by Aerts (2015) and Widen (2009).

The behavioral model used in our study was based on the work of Richardson (2010) (CREST model) because it is simple to implement and can be validated. It takes into consideration the number of individuals in the household as well as the type of day (weekday/weekend) to determine the presence and activity profiles. It is based on a stochastic method (Markov chain), which generates the presence profiles of a household. A transition matrix is established based on TUS and, at 10minute intervals, it generates the presence probability for households of 1 to 5 individuals while allowing variation in the number of persons present. The activity profiles for weekdays and the weekend are also calculated every 10 minutes based on the number of individuals in the house. To adapt the CREST model to the building stock in the Province of Quebec (Canada), we used data on TUS for the years 2011 and 2015 from Statistics Canada (2011) (2015) to calculate the start states and the transition matrices for the presence and activity profiles.

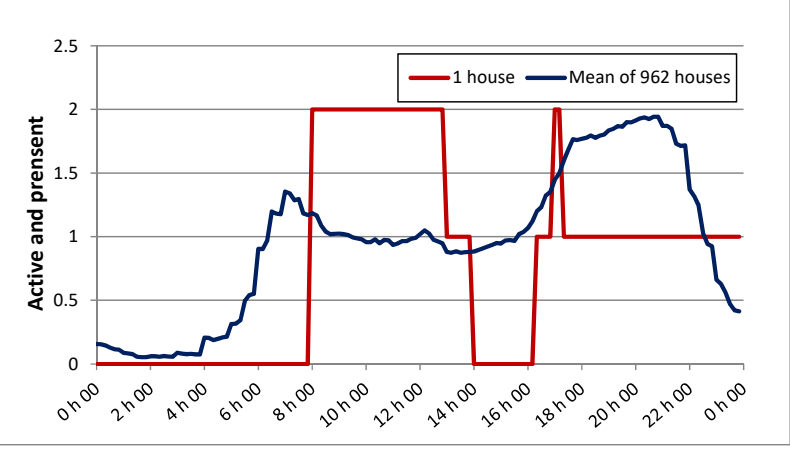

Figure 5: Number of active and present persons (January 2)

For example, Figure 5 shows the profile of the number of individuals present carrying out activities. The curve in blue presents the mean profile for all of the 962 houses of the simulated stock, whereas the curve in red presents the profile for a randomly selected house among the building stock. The figure shows that, from midnight to 6 a.m., no one is active, whereas in the afternoon, two individuals are active. For this specific simulated stock, the number of active individuals is greater in the morning and evening and, logically, lower during the night.

The main disadvantage of this model is that its presence profile only takes into account the active individuals in the building because it applies to the entire household and not to each individual. Furthermore, the proposed approach does not consider the duration of an activity or the arrivals and departures of the occupants to determine the activity profiles.

\section{Electricity consumption of appliances}

The activity model shown previously provides the use profiles of household appliances. Based on this information, we can determine the electric consumption profiles of those appliances, as presented below.

\section{Space heating devices}

Electric baseboards are the main heating system in $66 \%$ of Quebec dwellings, according to NRCan (2014). The model considers each room as a specific thermal zone heated by a baseboard. Vivian (2017) argues that a grey box model with two thermal masses is suitable to assess the impacts of temperature settings in each zone and correctly estimate peak demand. A model was developed considering that finding.

$$
\begin{gathered}
M C \frac{d T}{d t}=U A\left(T_{o}-T\right)+U 1(T 1-T)+A_{s} R_{s}+G+Q \\
M 1 \frac{d T 1}{d t}=U 1(T-T 1)
\end{gathered}
$$

where:

$M C(\mathrm{Wh} / \mathrm{C})$ and $U A(\mathrm{~W} / \mathrm{C})$ are respectively the "light" thermal mass and the heat loss coefficient between indoor and outdoor temperatures $T$ and $T_{o} ; M 1$ and $U 1$ are the "heavy" thermal mass and the internal heat transfer coefficient toward that mass with temperature $T 1 ; A_{S}\left(\mathrm{~m}^{2}\right)$ is the solar aperture; $R_{S}\left(\mathrm{~W} / \mathrm{m}^{2}\right)$ is the solar radiation; $G$ and $Q(\mathrm{~W})$ are the heat gains (metabolic and equipment) and the heating power, respectively.

Electronic thermostats are emulated by reducing the heating power when the temperature $T$ of the zone approaches the thermostat setpoint. Temperature settings, including setback schedules, were stochastically reproduced in the model based on occupant behaviors reported in surveys (Léger, 2003; Saine, 2010).

This model was validated based on the data of 10 instrumented houses (Fournier, 2018) where the electric power for heating and appliances, as well as setpoint and room temperatures, were monitored at one-minute intervals over several years. Figure 6 shows the total electric heating demand of one of these houses during one week in January 2017. The simulation was performed using local weather data. All other electric devices were considered to be sharing heat gains with specific zones. The observed discrepancies are partly related to the heat gains, which are instantly transferred to the thermal mass $M C$.

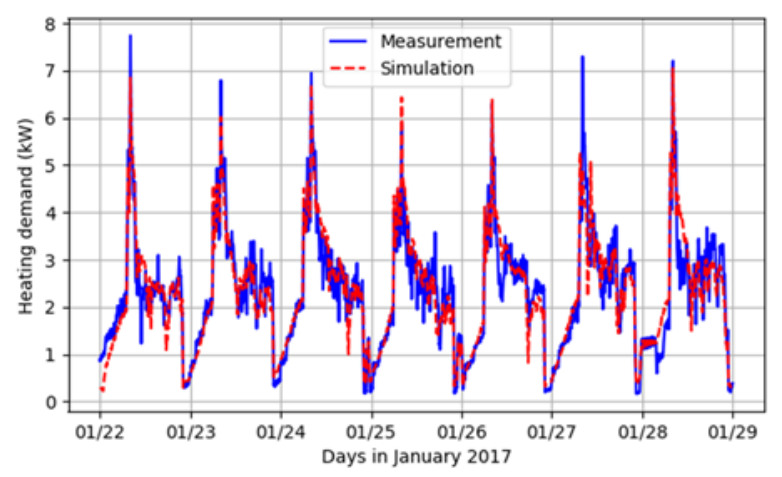

Figure 6: Electric heating demand profiles 
Since the model is used to predict trends in existing building stock, historical data can be used for its implementation. In this context, the overall heat loss coefficient $\left(\mathrm{W} /{ }^{\circ} \mathrm{C}\right)$ of a modeled electrically heated house is derived from the regression of daily meter readings against the local daily outdoor temperature. This coefficient is then split to the thermal zones in proportion with the floor area. The other parameters of the zones $\left(M C, U 1, A_{S}\right)$ are obtained through correlation with the heat loss coefficient except the rated heating capacity, which, as indicated previously, is related to the zone types and floor area.

\section{Hot water consumption}

Using a survey of 600,000 customers between 2002 and 2010 along with data from NRCan (2014b), the hot water consumption by occupant and by appliance was determined. Based on this information, a calibration of the activation rate, as well as hot water consumption by appliances (washing machines, dishwashers) and other uses (baths, showers, etc.) is integrated into the CREST model. The aggregated hot water use profile of the household then feeds the water heater model.

\section{Water heaters}

Most Quebec houses are equipped with a storage electric water heater. This device is modeled as a single water volume heated by an electric element located at the bottom of the tank. A thermostat keeps the water at $60^{\circ} \mathrm{C}$, while the heat loss from the tank is part of the heat gain in the zone. Two tank sizes are modeled: 180 and 270 litres of 3,000 or $4,500 \mathrm{~W}$, respectively.

\section{Household appliances}

Some major appliances are modeled in detail (refrigerators, dryers, electric ranges). In 10 occupied houses, the consumption of these appliances was metered at one-minute intervals. The profiles were divided into use events. Representative consumption profiles were deduced and included in the model. A profile is recalled each time the appliance is activated by the occupant, or on a periodic basis such as the refrigerator in Figure 7. In this case, a sequence of cooling and defrosting patterns is repeated, incorporating the randomness observed into the measured data.

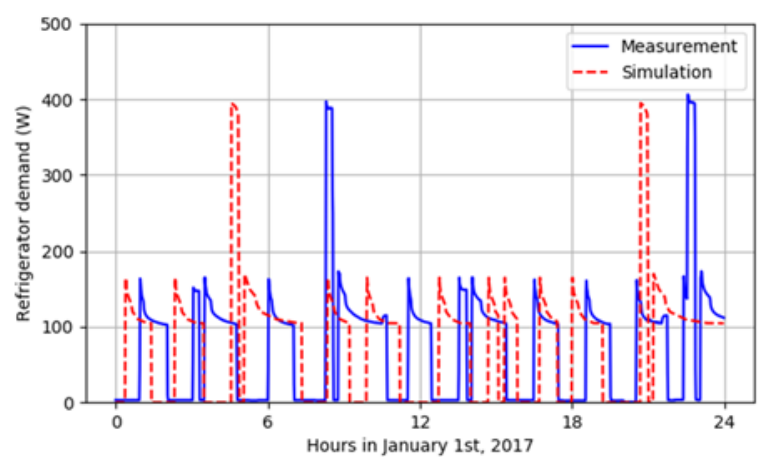

Figure 7: Refrigerator demand profile

Other appliances

Other appliances such as washing machines, dishwashers, home electronics, and small cleaning or cooking devices were modeled with a probability of activation, power level and duration of use, like in the CREST model approach. As reported by Wills (2017), a baseload must be added in order to include appliances that are not considered in the model. In our study, a baseload of about $500 \mathrm{~W}$ was assumed, which should be reduced as new appliances are added to the model. The heat dissipated by these appliances is added to the heat gain in the zones.

Lighting

Lighting is treated as in Fisher (2015) or Widen (2009), who propose to correlate lighting with the daylight level and the number of active occupants in the household.

\section{Calibration}

A similar calibration approach to Richardson's (2010) was applied in this study. A calibration scalar was used to represent the rate at which electrical devices begin to operate, based on the occupation model. For example, Figure 8 shows the power demand profile of an electric range for one house (red) and for the entire stock of 962 houses (blue) by applying this calibration scalar to the cooking activity.

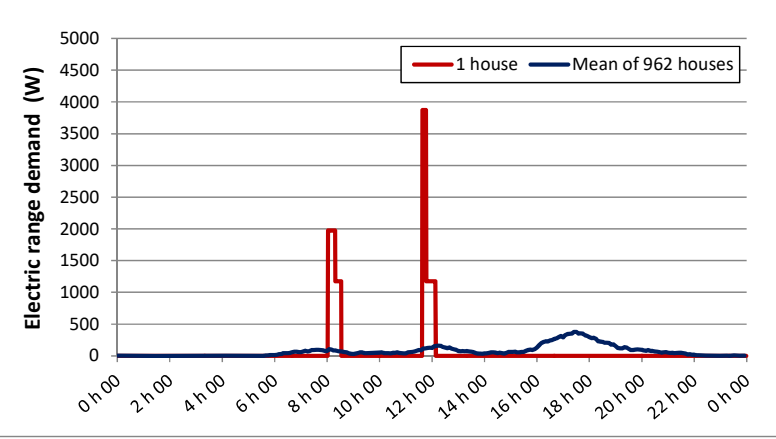

Figure 8: Electric range demand profile (January 2)

The calibration scalar was calculated so that annual energy and hot-water consumption corresponded with the country-wide data provided by NRCan (2018). Figure 9 presents the results obtained for the energy consumed by the main electrical appliances in a housing stock of 962 dwellings. These results are similar to the NRCan (2018) data and demonstrate that there is still room for improvement where several appliances are concerned.

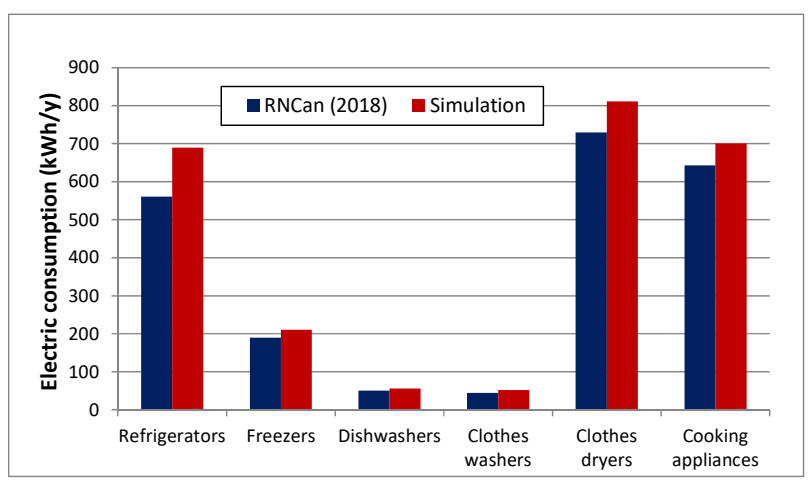

Figure 9: Annual electricity consumption of appliances 


\section{Preliminary Results}

The proposed methodology was applied to customers of an electric utility supplied by a distribution substation in a neighborhood of Montreal, Quebec, where temperatures are below $0^{\circ} \mathrm{C}$ for about half of the year. 962 residential customers supplied by this substation were selected based on criteria such as living in single detached homes and high probability of the home to be heated solely by electricity. A regression between the total daily electric consumption and daily outside air temperature (OAT) had been previously calculated for each customer to verify this last criterion. Anonymized 15-minute consumption profiles for year 2015 were used for the study. The aggregation of the 962 electric profiles measured during this period was the reference used in order to qualify the preliminary results of this approach.

Inputs for the model for each of the 962 dwellings were stochastically obtained from the Bayesian network explained in a previous section. Some known attribute values, such as square footage and dwelling type, have been used as constraints in the Bayesian network. Simulations of all electrical appliances in all dwellings were carried out over the year 2015 at 1-minute intervals, and the profiles were aggregated. To ensure that the stochasticity of the inputs did not impact the obtained aggregated profile, several sets of simulations were rerun and the aggregated simulated profiles were compared. As expected, due to the relatively high number of dwellings in the dataset no significant change was observed.

The average daily power demand per dwelling against the daily OAT for both measured and simulated aggregated profiles is depicted in Figure 10. The slope of consumption when the OAT gets colder is relatively the same between the simulated and measured profiles. However, simulated consumption is higher than measured consumption during the cold season. This over-prediction was attributed to a poor representation of solar heat gains and overestimated below-grade heat losses. Inversely, simulated consumption is lower than measured consumption during the summer months (OAT $>20{ }^{\circ} \mathrm{C}$ ). This could be easily explained by the fact that equipment such as air conditioners and pool pump filters were not included in the model. The development of these equipment models has not been prioritized because the peak demand, which is an important input in grid distribution planning, occurs in the winter.

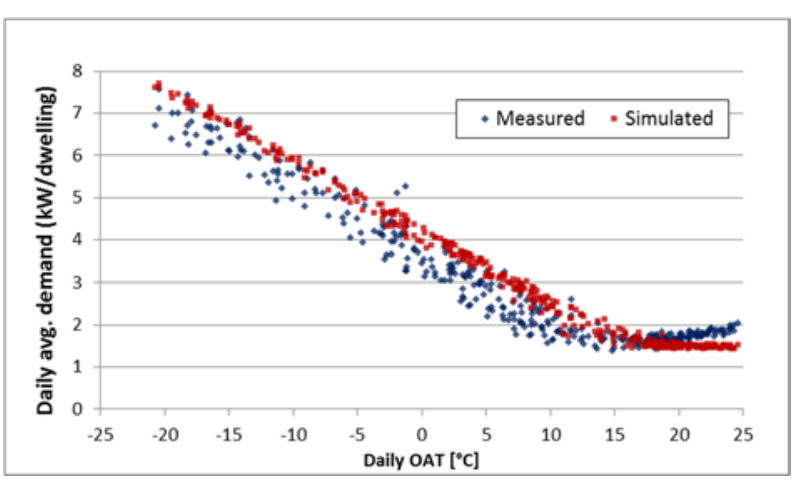

Figure 10: Daily average power demand vs daily OAT

The working daily measured and simulated profiles for January, November and August are shown in Figure 11. The simulated peak demand for January weekdays is close to the measured one, but with an offset of about an hour. This offset could be attributed to many behavioral aspects, such as temperature setpoint strategies and the offset between starting activities, appliance use and hot water draw. The simulated profile for November overestimates the measured profile relatively constantly throughout the day. As expected, in August, the trends in the measured and simulated profiles are different, especially at midday, due to missing appliances.

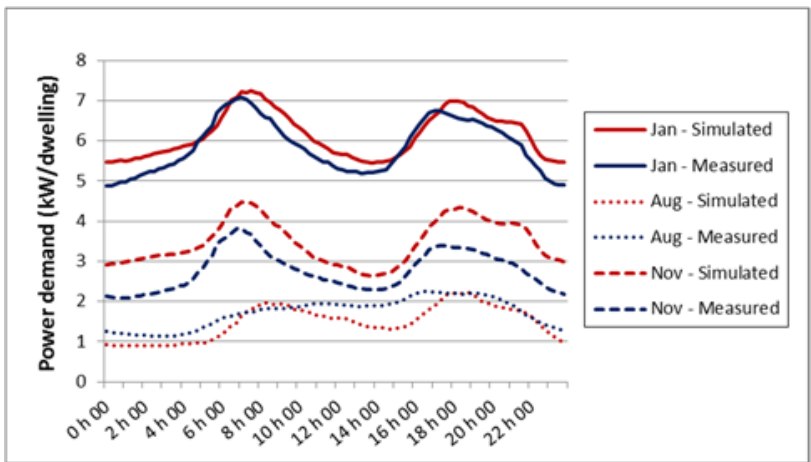

Figure 11: Daily profiles for weekdays in January,

November and August, both measured and simulated

One of the most interesting aspects of this kind of model is that it captures the contribution of each usage or appliance to the load. Figure 12 shows the stacked profile of major appliances/usage for a relatively cold day (January 9, 2015). As shown, electric heating contributed the most to the power demand. The water heater was in phase with morning and evening peak demand. Space heating also contributed to peak demand due to heat pickup after the overnight temperature setback ends early in the morning. 


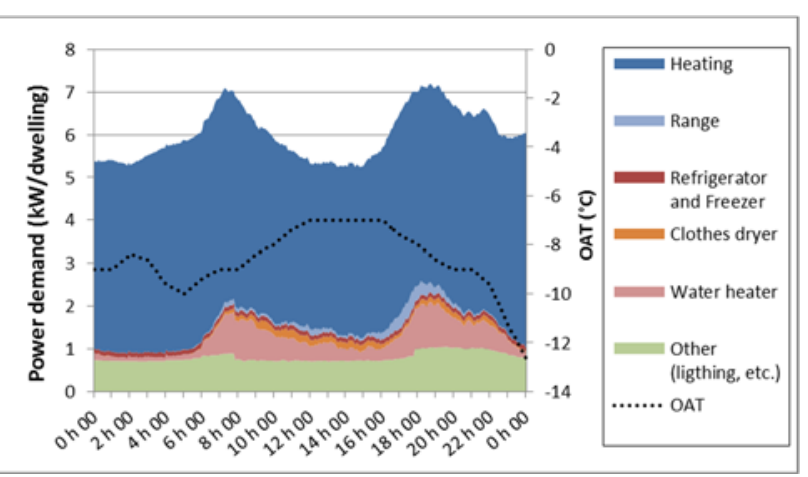

Figure 12: Power demand per usage for Jan. 9, 2015

Figure 13 shows the interquartile range (IQR) data points of both the simulated and measured profiles for several days in January. The IQR is slightly wider in the measured data compared to the simulated data, which indicates that some statistical variability is not captured by the model. At this stage, the model and the individualized measures for each home could be significantly different considering the stochastic attribution of several parameters. It has been demonstrated that the accuracy of the model improves with an increased number of aggregated profiles. Since the objective of the project is to model electricity demand at the connection point (transformer) linking a small number of dwellings, it is essential that the error be accurately determined and to use as much information as possible to increase the representativeness of the model on a small scale.

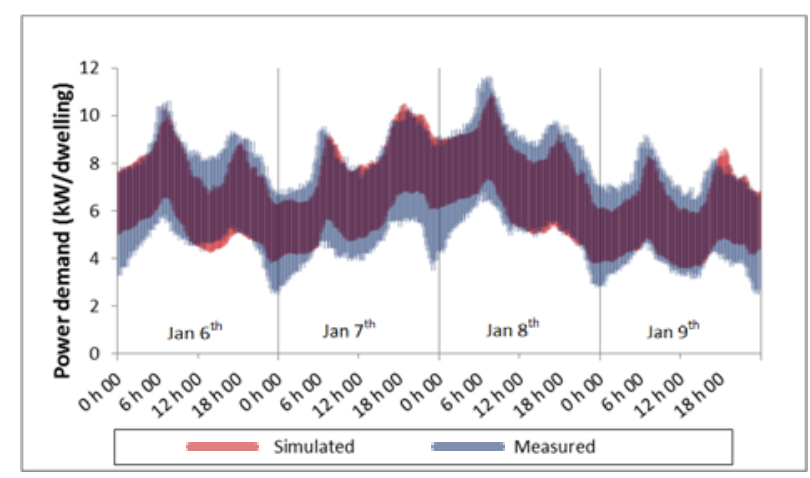

Figure 13: Interquartile range of 962 simulated and measured aggregated profiles for 4 days in January 2015

\section{Future work}

Many improvements to the model are planned. The preliminary step was to establish a proof of concept concerning the approach. With little calibration, the preliminary results are promising in terms of forecasting the load of a group of dwellings while taking into account the individual impacts of electrical appliances and the behavioral effects of occupants.

New appliance models, including air conditioners, swimming pool filter pumps, and pool and spa heaters, will be incorporated into the model. One of the challenges in the development and improvement of appliance models is to get representative data on electricity consumption and hot water draws in order to calibrate the model.
Since electric heating predominates in the contribution to peak demand, it is critical to improve the estimation of electrical heating, for example by considering interzonal heat transfer and giving better consideration to solar gains and heat losses to the ground. In the short term, the model should take into account the other main heating systems in Quebec (central forced air, central water, heat pumps) as well as fossil fuel systems, whether they be the main or secondary heat supply. In addition, multiunit buildings should be integrated into the model.

The heart of the model is the evaluation of occupant behavior. The CREST behavioral model used in this proof of concept is based on use of appliances, rather than behavioral patterns or activities of individuals. It is therefore difficult to evaluate the impact of changes in the individual behavior on the use of appliances. This is an important element for the continuation of our work, which will focus on forecasting the impact of a rate signal during critical peak periods affecting the load curve of a group of households. A new behavioral model based on the approaches of Wilke [2013] and Vorger [2014] will be developed to deal with the limitations of the CREST model and to take into consideration seasonal variations in behavior. Since the power consumption of electrical appliances is highly dependent on the behavioral model, appliance model calibration will have to be undertaken again once the new model is implemented. This will require determining the attributes of the individuals who have the highest impact on presence and behavioral factors. Consequently, the Bayesian network currently used to determine the attributes of the dwellings, households, individuals and appliances will have to be adapted to the new attributes identified.

\section{Conclusion}

A vision of a stochastic model of residential electric loads using a bottom-up approach was presented in this paper and applied to a set of 962 electrically heated homes. The final objective of the model is to predict the load profile on a regional scale under different scenarios of evolution for distribution network planning, which requires spatial and temporal patterns of power demand in the region concerned. This method is highly dataintensive for characterizing buildings, defining occupant behaviors/activities and implementing appropriate models for a large number of appliances. However, aggregating each load, which depends on household activities, gives a deep understanding of the demand profile and extrapolates new conditions (technologies and/or behavior) more easily compared to the usual load forecasting method.

\section{Acknowledgments}

This analysis is based on Statistics Canada Product62M0004XCB from the Survey of Household Spending for the reference year 2009 and on the General Social Surveys for the reference years 2011 and 2015. All computations, use and interpretation of these data are entirely those of the authors. 


\section{References}

Aerts, D. (2015). Occupancy and activity modelling for building energy demand simulation, comparative feedback and residential electricity demand characterisation. Thesis. Vrije Universiteit Brussel.

Ansanay-Alex, G., Abdelouadoud, Y. and Schetelat P. (2016). Statistical and Stochastic Modelling of French Households and Their Energy Consuming Activities. 12th REHVA World Congress - CLIMA 2016.

Capasso, A., Grattieri, W., Lamedica, R. and Prudenzi, A. (1994). A bottom-up approach to residential load modeling. IEEE Transaction on power system 9. 957-964.

Fischer, D., Härtla, A. and Wille-Haussmann, B. (2015). Model for electric load profiles with high time resolution for German households. Energy and Buildings 92. 170-179.

Fournier, M., Leduc, M., Sansregret, S. and Poulin, A. (2018). Making the Connection: Testing LineVoltage Communicating Thermostats for Baseboard Heaters in DR and EE Experiments, 2018 ACEEE Summer Study on Energy Efficiency in Buildings, Aug 2018.

Happle, G., Fonseca, J.A. and Schlueter, A. (2018) A review on occupant behavior in urban building energy model, Energy \& Buildings 174, 276-292

Kuster, C., Rezgui, Y. and Mourshed, M. (2017). Electrical load forecasting models: a critical systematic review. Sustainable Cities and Society 35. 257-270.

Léger Marketing (2003). Habitudes d'utilisation des thermostats, June.

Natural Resources Canada (NRCan) (2014b). Energy Consumption of Major Household Appliances Shipped in Canada. Trends for 1990-2011.

Natural Resources Canada (NRCan) (2014a). Survey of Household Energy Use 2011, Detailed Statistical Report.

Natural Resources Canada (NRCan) (2018). Energy Use Data Handbook 1990-2015. Handbook.

Paatero, J.V. and Lund, P.D. (2006). A model for generating household electricity load profiles, International Journal of Energy Research 30. 273290.

Page, J., Robinson, D., Morel, N. and Scartezzini, J.L. (2008). A generalised stochastic model for the simulation of occupant presence, Energy and Buildings 40. 83-98.

Richardson, I., Thomson, M. and Infield, D. (2008). A high-resolution occupancy model for energy demand simulation. Energy and Buildings 40. 1560-1566.

Richardson, I. Thomson, M. Infield, D. Clifford C. (2010). Domestic electricity use: A high-resolution energy demand model. Energy and Buildings 42. $1878-1887$.

Saine Marketing (2010). Étude sur les habitudes et comportements des clients résidentiels envers l'efficacité énergétique - Édition 2010, Final Report, September.

Statistics Canada (2016). Census. Data tables.

Statistics Canada (2009). Survey of Household Spending. Microdata File.

Statistics Canada (2011). General Social Survey. Cycle 24: Time-Stress and Well-Being. Microdata File.

Statistics Canada (2015). General Social Survey. Cycle 29: Time Use. Microdata File.

Vivian, J., Zarrella A., Emmi, G. and De Carli, M. (2017) An evaluation of the suitability of lumpedcapacitance models in calculating energy needs and thermal behaviour of buildings, Energy and Buildings 150. 447-465.

Vorger, E. (2014). Étude de l'influence du comportement des occupants sur la performance énergétique des bâtiments. Thesis. Ecole nationale supérieure des Mines de Paris.

Widén, J. and Wäckelgård, E. (2009). A high-resolution stochastic model of domestic activity patterns and electricity demand. Apply Energy 87. 1880-1892.

Wilke, U. (2013). Probabilistic Bottom-up Modelling of Occupancy and Activities to Predict Electricity Demand in Residential Buildings, Thesis, École Polytechnique Fédérale de Lausanne.

Wills, A.D., Beausoleil-Morrison, I. and Ugursal, V.I. (2017). Adaptation and validation of an existing bottom-up model for simulating temporal and interdwelling variations of residential appliance and lighting demands. Journal of Building Performance Simulation 11(3). 350-368. 